\title{
GESTIÓN PÚBLICO PRIVADA, DESARROLLO E INSERCIÓN INTERNACIONAL. EL POLO INFORMÁTICO DE TANDIL
}

\author{
Micaela BARrios \\ Universidad Nacional de Quilmes, Argentina \\ barriosmicaela88@gmail.com \\ Ignacio De Angelis \\ Universidad Nacional del Centro, Argentina. \\ deangelisignacio@gmail.com
}

\begin{abstract}
RESUMEN
El artículo analiza la dinámica de articulación público privada en torno al surgimiento y consolidación del Polo Informático en la ciudad argentina de Tandil. La hipótesis que guía el trabajo sostiene que el desarrollo local del sector de Software y Servicios Informáticos superó su ponderación inicial como modelo de negocio y prosperó hacia un proyecto de desarrollo estratégico para la ciudad y su inserción internacional. Los hallazgos muestran que la interacción lograda entre la universidad, el municipio y las empresas, permitió el impulso competitivo del sector, promoviendo al mismo tiempo a Tandil como una marca de exportación de software y servicios tecnológicos de calidad.
\end{abstract}

Palabras claves: Desarrollo local, Asociaciones público-privadas, Inserción internacional subnacional, Argentina. 


\title{
PUBLIC-PRIVATE MANAGEMENT, DEVELOPMENT AND INTERNATIONAL INSERTION: THE TANDIL INFORMATION TECHNOLOGY POLE
}

\begin{abstract}
The article analyzes the dynamics of public-private articulation around the emergence and consolidation of the information technology pole in the city of Tandil, Argentina. Its hypothesis asserts that the local development of the software and IT services sector exceeded its initial weighting as a business model and prospered to become a strategic development project for the city and its international insertion. The findings show that the interaction achieved between the university, the municipal government and companies enabled the sector to gain in competitiveness, whilst also promoting Tandil as a brand for the export of high-quality software and technological services.
\end{abstract}

Keywords: Local development, public-private partnerships, sub-national international insertion, Argentina. 


\section{INTRODUCCIÓN}

Históricamente, la ciencia económica ha considerado a los servicios como una actividad no transable y de baja productividad. Dicho enfoque hoy ha tomado un rumbo distinto, ya que, actualmente, se desarrollan numerosas actividades de servicios de alta transabilidad internacional y de una creciente productividad. En este marco, se sitúan los denominados servicios basados en conocimiento ${ }^{1}$, los cuales se caracterizan por ser intensivos en trabajo calificado y por el uso y desarrollo de tecnologías avanzadas para su prestación (López, Niembro y Ramos 2011). En tanto que el software y los servicios informáticos son considerados una actividad económica de los servicios basados en el conocimiento.

A nivel mundial, el fenómeno de la globalización junto con el avance de las tecnologías de la información y la comunicación permitió el desarrollo y la profundización de la prestación de servicios de manera remota, de forma barata y confiable. Asimismo, los actuales patrones demográficos y de consumo, sumado a los procesos de liberalización y deslocalización de la producción, permitieron una gran apertura de los mercados junto con una mayor integración económica global. Situación que, además de traer a escena a países con enormes reservas de mano de obra, como son los casos de China e India, dio lugar a nuevas y renovadas oportunidades de negocios globales.

Como resultado, la línea que hoy divide las manufacturas y los servicios se torna menos clara. Por ejemplo, cuando una empresa como Apple introduce en el mercado su iPad, un producto manufacturado, este involucra un gran componente de servicios, no solo en la etapa de comercialización, sino también en las etapas previas y post venta. En efecto, la contribución de diversos servicios ofrecidos a los clientes de Apple agrega un importante valor al producto, ya que los mismos pueden acceder a servicios que la empresa ofrece para mejorar la interacción y la experiencia del usuario (Kraemer, Linden y Dedrick 2011).

Al respecto, Jensen (2009) asegura que desde la década de los noventa, se ha redireccionado el offshoring ${ }^{2}$ de los productos manufacturados a los

1 Los servicios basados en el conocimiento son aquellos servicios que emplean intensivamente en su producción un alto contenido científico y tecnológico y, que necesitan ser brindados por mano de obra calificada. Abarcan servicios financieros, de salud, inmobiliarios, de ingeniería, contables, de publicidad, de software e informáticos, audiovisuales, empresariales, profesionales y técnicos, consultoría en general e investigación y desarrollo (Blanco 2016).

2 Se denomina offshoring al proceso de outsourcing, entendido este último como aquella parte de la actividad productiva desarrollada mediante medios externos a la empresa, 
servicios, dado que cada vez se requieren servicios más avanzados en áreas técnicas y administrativas. La utilización de centros de servicios offshore para temas tecnológicos, de gestión, de ingeniería y de contenidos audiovisuales, son parte constitutiva de las nuevas redes globales de producción (Scholvin 2020).

La deslocalización de actividades fue variando desde sectores mano de obra intensivos de bajo valor agregado hacia actividades intensivas en conocimiento. La demanda fue impulsada mayormente por los requerimientos de las empresas de los países centrales y luego se extendió hacia países emergentes, fortaleciendo la localización de firmas especializadas en los países de América Latina. En efecto, la industria de servicios offshore permitió a los países de la región diversificar su inserción internacional y escalonamiento en las cadenas globales de valor, generando empleo calificado e ingresos de divisas aún en contextos recesivos (Fernandéz Stark, Bamber y Gereffi 2011).

En Argentina, las exportaciones de este tipo de servicios crecieron notablemente desde comienzos del nuevo siglo. La salida devaluatoria de la convertibilidad abarató los costos de la mano de obra, seguido por un cambio en el ciclo del crecimiento y recomposición productiva, junto con el establecimiento de una estrategia de desarrollo y promoción de áreas estratégicas, entre las cuales se encuentra la industria del software (De Angelis 2017). El principal mecanismo promocional consistió en el establecimiento de un régimen fiscal especial para el sector, con importantes beneficios para las empresas que tienen como actividad principal el desarrollo de software o la provisión de servicios informáticos, Ley 25.922 (Gajst 2012).

En este marco, el boom del offshoring de servicios con alto contenido tecnológico produjo el alza de la demanda (Tamayo Plata 2014), permitiendo que distintas ciudades de Argentina comiencen a ser elegidas como un destino apto para deslocalizar la prestación de servicios comerciables internacionalmente.

Durante el año 2017, en Argentina el sector de software y servicios informáticos generó US\$2.237.000.000 de ventas en el mercado interno y US\$ 1.699.000.000 en exportaciones, al tiempo que, en términos de

en la misma zona geográfica o en otra, pero que en el caso del offshoring implica el traslado al extranjero de los medios de producción necesarios para llevar a cabo esa actividad, ya sea si los medios de producción se mantienen internos en la empresa o si son terciarizados (CDTI 2007). 
empleo, se alcanzó la cifra de 107.100 profesionales de software (OPSSI 2018).

Teniendo en cuenta estas consideraciones, el artículo se centra en analizar la conformación y desarrollo del Polo Informático en la ciudad de Tandil como una iniciativa del sector público, impulsada inicialmente por la Universidad Nacional del Centro de la Provincia de Buenos Aires (Unicen), que permitió dotar al territorio de una estrategia de desarrollo local y de vinculación comercial internacional. Es decir, la experiencia superó la consideración como modelo de negocio, evolucionando hacia una concepción estratégica amplia para el desarrollo socioeconómico local.

Albuquerque (2002) señala que el desarrollo local debe entenderse como un proceso participativo dentro del territorio, impulsado por la cooperación entre actores para el establecimiento de una estrategia de desarrollo común. Asimismo, este proceso tiene como objetivo "estimular la actividad económica y crear empleo de calidad, a partir del aprovechamiento de los recursos y potencialidades locales, y las oportunidades derivadas del contexto global" (Albuquerque 2002: 15). Se plantea así, como un agregado de valor de los gobiernos locales la capacidad de establecer la arquitectura y proceso social necesario para incentivar, coordinar y orientar dicha cooperación.

Desde la perspectiva de la inserción internacional del desarrollo local se ha destacado el rol histórico pero cada vez más importante las ciudades como espacios globales (Taylor 2007, Taylor y Darudder 2016). Siguiendo a Taylor, la construcción de una red global de ciudades impulsada por la interconexión de firmas de servicios líderes a nivel global, trasciende en la actualidad a las grandes ciudades, fortaleciendo las relaciones entre ciudades de distintos tamaños y generando una red cada vez más en la generación de innovación y desarrollo.

En la teoría de las ciudades globales se destaca el impulso de las empresas de servicios para la conformación de las redes de interconexión (jurídicos, financieros, publicitarios y, más recientemente, el sector de software y servicios informáticos). En este marco, los servicios asociados al software se presentan como un enclave dinámico para la inserción de ciudades de menor importancia relativa, redefiniendo su localización y su posicionamiento en la trama productiva global.

En el plano local, las redes de colaboración entre asociaciones, empresas, universidad y el gobierno, componen también un mecanismo esencial para 
el uso eficiente de los recursos disponibles localmente y para mejorar la competitividad territorial (Cravocuore 2006).

En efecto, como se analiza a lo largo del artículo, la creación del Polo Informático en Tandil surgió como parte de una iniciativa propuesta por la universidad a partir de distintos convenios con empresas de software y servicios informáticos con el fin de que se instalaran en la ciudad. Por su parte, el Municipio acompañó esta idea como segmento de una nueva estrategia de desarrollo local para la ciudad y la región, la cual permitió que Tandil hoy tenga una proyección comercial nacional e internacional distintiva, dinámica y novedosa para una ciudad de tamaño intermedio.

El Polo Informático de Tandil hoy emplea 1.800 personas, está conformado por cerca de 60 empresas y exporta el $70 \%$ de lo que produce a nivel local, constituyéndose como uno de los sectores de mayor crecimiento, potencial e inserción comercial internacional de la zona centro de la Provincia de Buenos Aires. De esta manera, el presente trabajo aborda su estudio como antecedente pertinente para la implementación de estrategias de desarrollo y modelos de negocio en ciudades de tamaño intermedio con proyección global.

Con todo, en base a una metodología fundamentalmente cuantitativa, el relevamiento de datos y la realización de entrevistas, el trabajo analiza el desarrollo y evolución del sector de software y servicios informáticos a nivel local, reconociendo como hipótesis de trabajo, que su conformación tuvo lugar por el impulso inicial de la Unicen y, luego, con la consolidación de las distintas firmas, logró consolidarse a partir de la iniciativa y el liderazgo del sector privado, acompañado por la universidad y el municipio como socios estratégicos, cuya sinergia generó un dinámico ecosistema de cooperación, crecimiento y desarrollo para el sector y el territorio que lo contiene.

En cuanto a la organización del texto, en primer lugar, presenta la ciudad de Tandil y sus principales características socioproductivas. En el segundo lugar, se analiza el proceso de surgimiento del Polo Informático y el rol de la Unicen en la construcción de la infraestructura del conocimiento requerida para su consolidación. Como tercer punto se explica el rol del gobierno municipal y los proyectos estratégicos de desarrollo propuestos que incluyen al sector de software y servicios informáticos local. Por último, el artículo aborda el análisis del sector de software y servicios informáticos en Tandil, sus características principales, el impacto socioeconómico en la ciudad y sus perspectivas futuras de negocio. 


\section{TANDIL: ESTRUCTURA SOCIO-PRODUCTIVA E INSERCIÓN INTERNACIONAL}

La ciudad de Tandil constituye un Municipio de tamaño intermedio con alrededor de 130.000 habitantes. Se encuentra localizada en el centro de la provincia de Buenos Aires, a menos de 400 kilómentros de Capital Federal y cuenta con un Índice de Desarrollo Humano (IDH) de 0,89 , situándose dentro del conjunto de los más altos de la provincia (De Angelis y Lorenzo 2016). Asimismo, se ubica en el puesto 24 en el ranking del Índice de Bienestar elaborado por Velázquez et al. (2010), sobre un total de 528 ciudades nacionales, siendo considerada como una de las ciudades con mejor calidad de vida del país.

Tandil forma parte de una región clasificada como fundamentalmente agropecuaria (MEPBA 2014), pero que, a su vez, se caracteriza por su diversidad productiva. En la estructura económica, la industria manufacturera tiene una participación aproximada del 20\%, con una destacada presencia histórica de la industria metalúrgica y metalmecánica, la agroindustria y la industria alimentaria, sobresaliendo la industria láctea con más de 150 tambos en actividad. Cuenta, a su vez, con un desarrollado y significativo sector de agricultura, ganadería, caza y silvicultura con una participación cercana al 15\% (Calvento et al. 2016).

Geográficamente, la ciudad se caracteriza por un entorno de llanura y a su vez de sierra. Esta combinación genera un paisaje único y natural que permitió a Tandil desarrollarse turísticamente. En este sentido, se ha desarrollado una importante evolución del sector terciario, fundamentalmente las actividades asociadas al comercio, hoteles, restaurantes y servicios inmobiliarios, que en conjunto explican alrededor del 25\% del producto total. En tanto que los servicios y la administración pública tienen un impacto cercano al $17 \%$ en la actividad económica local (De Angelis y Lorenzo 2016).

Desde su nacimiento en 1974, la Universidad Nacional del Centro constituye uno de los inductores principales de desarrollo y progreso económico local. La institución tiene un perfil de formación vinculado con las necesidades la región socioeconómica de la comunidad local. En la década de los ochenta, Tandil comenzó un proceso de crecimiento debido a la conjugación de tres factores: el arribo constante de estudiantes universitarios de otras localidades, el crecimiento productivo y turístico del partido, y de manera vinculada, las inversiones de los productores agrícolas, que adquirían inmuebles con las ganancias generadas por la producción de soja (Capellán 2016). Este crecimiento se mantuvo constante durante 
la década del noventa y, a partir del año 2000, adquirió características distintivas de un proceso de crecimiento acelerado superior a la media (Finquelievich, Feldman y Girolimo 2017).

En este marco, Tandil adquirió un perfil productivo asociado al crecimiento de los servicios, los cuales explican la existencia de casi el 80\% de las empresas locales y el $70 \%$ de los puestos de trabajo (Calvento et al. 2016). Por un lado, se destaca la relevancia creciente del turismo y las actividades asociadas, y de forma más reciente, como consecuencia de la creación del Polo Informático en 2003, se encuentran los servicios de software y servicios informáticos.

Con respecto a su proyección e inserción internacional, a partir del año 2003 el municipio de Tandil se comprometió a fomentar una mayor internacionalización del territorio mediante la definición y reorientación de la política exterior local. En este marco, en el año 2004 se creó la Oficina de Vinculación Internacional, cuyos objetivos claves son la búsqueda de cooperación internacional descentralizada, la acción para la integración regional y la promoción del comercio internacional. Dicha oficina trabaja en forma conjunta con la Secretaría de Desarrollo Local, que, junto a las mesas sectoriales de vinculación público-privado, se encarga de definir el rumbo de la política pública a implementar.

Asimismo, desde el año 2011 el municipio de Tandil desarrolla una política internacional subnacional enfocada en la participación de la Red de Mercociudades (la principal red de gobiernos locales del Mercosur), la realización de actividades vinculadas al comercio exterior y a la gestión de visitas protocolares de representantes diplomáticos extranjeros (De Angelis y Lorenzo 2016).

Para finalizar, se destacan las importantes acciones que el municipio ha realizado junto con otros actores locales para promocionar Tandil y generar nuevas inversiones, a partir de las cuales la ciudad es considerada referente de gestión pública innovadora por otras localidades (Finquelievich, Feldman y Girolimo 2017). Como resultado, el proceso de desarrollo distintivo de la ciudad, la vinculación público-privada que se lleva adelante, junto al el rol que ejerce la universidad en la comunidad local y regional, constituyen factores centrales para el desempeño socioeconómico y la obtención de resultados. 


\section{CONFORMACIÓN DEL POLO INFORMÁTICO EN TANDIL Y EL ROL DE LA UNIVERSIDAD}

Siguiendo a Becattini $(2002,2004)$ y su estudio de los distritos industriales, la especialización de los territorios en sectores específicos, con empresas integradas o arraigadas a la comunidad local, a sus normas y sus valores, permite generar factores específicos de competitividad. Estos elementos se encuentran asociados al flujo de la información, a la disponibilidad de proveedores y al rol de los actores institucionales (contacto con la Universidad y el gobierno local), lo cual permite que a partir de la cooperación entre actores tengan lugar procesos productivos flexibles, innovadores y cada vez más complejos.

En las décadas del ochenta y del noventa, la industria de software a nivel local solo tenía capacidad para resolver cuestiones propias de la ciudad. Desarrollar soluciones para necesidades provenientes de otro país era algo impensado, inclusive la exportación de software a nivel nacional solo se daba de manera excepcional (Taborga 2018). Por su parte, la universidad trabajaba en proyectos específicos de desarrollo con fuerte impronta de investigación. Los graduados de la carrera de Ingeniería en Sistemas, de la Facultad de Ciencias Exactas de la Unicen, optaban por migrar de la ciudad dado que no había un mercado local que pudiera absorberlos, ni tenían posibilidades de crecer profesionalmente.

Con el desarrollo del paradigma tecnológico de la información y la comunicación, la Unicen y sus grupos de investigación comenzaron a relacionarse y establecer mayores vínculos fuera del país, generando un importante nexo, aunque separado del sector empresarial. Mientras que, paralelamente, se fueron agrupando distintos profesionales del sector, pequeños emprendimientos, y empresas de menor tamańo, que brindaban principalmente soluciones en Tandil y la zona.

Paralelamente, como parte de una iniciativa propuesta por el rector de la Unicen a fin de encontrar solución al fenómeno de migración constante de graduados y la desconexión con el sistema productivo, acciones se puso en marcha un modelo institucional de apertura a la comunidad y de estrecha vinculación con la región. De esta manera, se plasmó la idea de generar un cluster que enarbolara los conceptos de gestión del conocimiento e interacción.

El Polo Informático de Tandil se creó en el año 2003 como un cluster sectorial del Parque Científico-Tecnológico de la Unicen y se enmarcó como eje central del Programa Institucional de Apoyo a la Actividad 
Productiva (PIAAP) de la Universidad, el cual se proponía justamente estimular la conformación de conglomerados productivos en áreas de actividad prometedoras implementando modalidades que permitieran y ayudaran al nacimiento de nuevas empresas de base tecnológica (Rébori, D’Annunzio y Dabós 2011).

En marzo del año 2003 la Unicen, como primer paso para crear el Polo y generar productos informáticos para la exportación, firmó un convenio con la empresa Idea Factory Software (IFS) del grupo BGH, con el objetivo de que esta última se instalara en el campus universitario de Tandil. A partir del convenio, la firma se comprometía a realizar una inversión inicial de $\$$ 2.000.000 y en principio en las oficinas de IFS en Tandil se desempeñarían diez ingenieros en sistema de la Unicen, contando con otros 1.000 que estarían disponibles en una base de datos para responder a la demanda.

Para el desarrollo del Polo se contó también con el apoyo desde el gobierno nacional y el provincial, que realizaron importantes inversiones en infraestructura para mejorar la conectividad a internet de la ciudad. Luego de la firma del convenio inicial, se suscribieron cerca de otros 50 convenios con empresas nacionales y multinacionales como Microsoft, Redimec, Accendra Networks, Mc Cain, Ábaco Informática, Snoop Consulting, Technisys, Unitech, entre otras.

Para que las empresas se instalaran en la ciudad y para facilitar su inserción dentro del Polo Informático, la Unicen les ofrecía un espacio físico y los servicios básicos para funcionar, la vinculación con los laboratorios de investigación ${ }^{3}$, con instituciones provinciales y nacionales y con otras empresas asociadas, servicios de consultoría y asistencia técnica y la búsqueda y contratación de personal (Rébori, D’Annunzio y Dabós 2011).

En efecto, la Unicen contaba con un importante stock de recursos humanos altamente capacitados, que mediante la Fundación Universidad Empresa (Funivemp) -cuyo objetivo era facilitar la articulación entre el sistema científico-tecnológico y productivo- ofrecía un circuito de pasantías que le permitía a las empresas acceder a una bolsa de empleo de la Universidad y poder contratar con una inversión inicial baja (Lopez Bidone 2008).

3 Se destaca el trabajo de los laboratorios Isistan (Instituto de Sistemas Tandil), INTIA (Instituto de Investigación en Tecnología Informática Avanzada) y Pladema (Instituto Plasmas Densos Magnetizados) como laboratorios de excelencia dentro de esa asociación pública-privada. 
En los inicios del Polo, muchas empresas comenzaron a funcionar en aulas del campus universitario, contando con pequeńos equipos de trabajo que se deslocalizaban de su sede central y se encargaban de formar un equipo local. La mayoría de las mismas fueron empresas de capitales argentinos con base en Buenos Aires y un pequeño grupo de trabajo en Tandil (cinco desarrolladores, aproximadamente), mientras que las empresas de capitales extranjeros tenían sede en Buenos Aires con anexos de trabajo en Tandil (Ribas, Dabós y Rivero 2007).

Durante los primeros años del Polo, la Unicen impulsó procesos de incubación de pequeños emprendimientos, colaboró con el armado de los planes de negocio, la obtención de financiamiento de distintos organismos, la vinculación con redes de inversores y la participación de las empresas del Polo en foros de capital de riesgo, entre otras acciones (Rébori, D’Annunzio y Dabós 2011). A medida que el Polo comenzó a crecer, las empresas demandaron otro tipo de asistencia por parte de la institución. Se puso de manifiesto la necesidad de contar con ámbitos propicios para la educación continua de los empleados y candidatos potenciales del sector de software y servicios informáticos. Como respuesta, la Unicen desarrolló, a través de programas de extensión, distintas capacitaciones y actualizaciones profesionales.

Por otro lado, durante esos años, se produjo un factor que impactó de manera directa en la evolución del sector. La ciudad comenzó un proceso de crecimiento de gran dinamismo, convirtiéndose en una ciudad atractiva por su calidad de vida para las capas medias. Esta situación le dio un importante impulso al Polo, motivó la repatriación de graduados y las empresas pasaron de tener un pequeño equipo de trabajo en Tandil a instalar una sede o un site en la ciudad, aumentando en consecuencia las ofertas laborales.

Un año después de la firma del primer convenio, el Polo Informático ya contaba con 23 empresas y 95 puestos de trabajo. En tanto que, para el cuarto año, el total de empresas ascendía a 53 con 450 puestos de trabajo activo. Como se analizará más adelante, el ritmo de creación de empresas con posterioridad a 2007 disminuyó, pero siguió mostrando gran dinamismo, ya que fueron creadas 21 firmas entre los ańos 2007 y 2017. Actualmente, cuenta con casi 60 empresas y se estima que para los próximos ańos la cantidad de empleos del sector alcance los 3.300. En este sentido, mientras que en la década del noventa Tandil retenía menos del 15\% de los jóvenes profesionales, hoy el Polo está en condiciones de absorber el $100 \%$ de los mismos. 
En síntesis, el rol de la universidad para la conformación y consolidación del Polo Informático fue fundamental y constituye un aspecto central de la dinámica de relacionamiento entre los actores. Asimismo, permitió el desarrollo de iniciativas trabajadas en conjunto, favoreció la competitividad de las empresas y promovió la cooperación entre las mismas.

\section{LA CÁMARA DE EMPRESAS DEL POLO INFORMÁTICO DE TANDIL}

En el año 2008 se creó la Asociación de Empresas del Polo Informático de Tandil (AEPIT) como resultado de la movilización de distintas empresas de software radicadas en la ciudad que apostaron a trabajar en conjunto, creando un ecosistema de colaboración orientado a desarrollar el sector y posicionar a Tandil como proveedor de software y servicios informáticos. En el año 2010, la AEPIT se transformó en la CEPIT (Cámara de Empresas del Polo Informático de Tandil), una asociación civil que trabaja en la vinculación y articulación entre el gobierno, las empresas y la universidad, con el objetivo de obtener mayores beneficios para las entidades participantes y para la comunidad local en general y, a su vez, alentar inversiones en la región.

Actualmente, la CEPIT agrupa a 50 empresas de software promoviendo un ambiente de coordinación entre las firmas y entre estas, la Unicen y el municipio, sus dos socios estratégicos. Su gestión es llevada a cabo por una comisión directiva formada por profesionales procedentes de las distintas empresas asociadas, elegidos en asamblea anual. Durante el año 2018 se establecieron los siguientes cinco ejes estratégicos en base a los cuales se organizaría la gestión de trabajo de la CEPIT hasta el año 2020 (CEPIT 2018):

- Formación de talento: el objetivo es preparar a los futuros. profesionales del sector. Al respecto la CEPIT proyecta incorporar 1.500 personas en el mercado laboral local.

- Especialización: cuyo fin es motivar e impulsar a las empresas en la generación de servicios y productos de mayor valor agregado en nuevas tecnologías (inteligencia artificial, lot, big data y blockchain).

- Emprendedorismo: se busca acompañar a los emprendedores en el proceso de desarrollo y consolidación de sus ideas hasta la creación de una empresa de base tecnológica.

- Modernización del Estado: cuyo objetivo es acompañar al municipio en la transformación digital del gobierno, asesorando y proveyendo servicios y productos. 
- Comercialización: busca potenciar la capacidad comercial y exportadora de las pymes del sector de software y servicios informáticos en un marco asociativo y de crecimiento conjunto.

El eje formación de talento es central en la gestión de CEPIT dado que la falta de recursos humanos capacitados para el sector de software y servicios informáticos es una de las principales problemáticas para las empresas en todo el país. Este eje está enfocado en tres actividades principales: el Plan 111 Mil (de alcance nacional) ${ }^{4}$, el Programa Click al Futuro ${ }^{5}$ y las tecnicaturas terciarias junto con la Unicen (ambas iniciativas de carácter local).

La CEPIT ofrece diferentes beneficios a sus asociados tales como oportunidades comerciales, capacitaciones, financiamiento, monitor estadístico y certificaciones. Con respecto a este último punto, actualmente se están realizando actividades asociativas para el proceso de certificaciones de calidad ISO y certificaciones de procesos de producción modelo de madurez y capacidad integrado ${ }^{6}$. En suma, la CAEPIT expresa la consolidación del sector empresario local. Actualmente, bajo su protagonismo, y con la Unicen y el municipio como socios estratégicos, lidera el Polo informático de Tandil, representando un ecosistema de cooperación, crecimiento y desarrollo a nivel local sin precedentes.

\section{EL GOBIERNO MUNICIPAL: SUS PROYECTOS DE DESARROLLO E INICIATIVAS DE APOYO AL SECTOR}

El municipio de Tandil como agente del desarrollo socioeconómico favorece la vinculación con actores locales, provinciales, nacionales e internacionales, 4 El Plan 111 Mil fue lanzado en 180 ciudades de todo el país. La CEPIT es la unidad ejecutora local desde 2017, con 40 instructores y 21 cursos en distintos puntos de la ciudad, uno en Azul y otro en Olavarría. En este sentido, Tandil lidera el listado de ciudades en las que el plan fue implementado por la cantidad de alumnos que lograrán certificar y por los que ya se encuentran contratados.

5 El programa Click al Futuro se enfoca en tres aristas. Por un lado, trabaja en conjunto con las instituciones educativas locales, en mejorar el sistema educativo mediante la incorporación de tecnología en todos los niveles escolares, por otro lado, de manera conjunta con la Unicen y el municipio, desde el ańo 2016 se realizan distintas actividades de difusión en colegios secundarios de las carreras en sistemas disponibles en la ciudad y del mercado laboral en auge que hay alrededor de ese tipo de carreras. La tercera arista es el Programa Avanzado de Educación Secundaria con énfasis en tecnologías de la información y la comunicación, más conocido como Escuelas PRO A (una nueva modalidad en colegios secundarios que apunta a la formación en tecnologías de la información)

6 El modelo de madurez y capacidad integrado, es un modelo de procesos que contiene las mejores prácticas de la industria para el desarrollo, mantenimiento, adquisición y operación de productos y servicios. 
a partir de herramientas de gestión relacional que fomentan iniciativas, espacios de articulación público-privada y colaboración multisectorial y que, a su vez, promueven políticas de innovación (Finquelievich, Feldman y Girolimo 2017). Cabe destacar que desde el año 2003, se mantiene el mismo gobierno a nivel local.

Durante los últimos años, se desarrollaron a nivel local distintas iniciativas y herramientas de política pública específicas para el sector de software y servicios informáticos. En el año 2009 se creó la Ordenanza Municipal de Fomento del Software y los Servicios Informáticos (Ordenanza 11681), mediante la cual Tandil se adhería a las leyes nacional 25.922 y provincial $13.649^{7}$ que promocionan la industria del software. A través de la ordenanza, aún vigente, se brindan a las empresas del sector, exenciones en el pago de las tasas municipales, estabilidad fiscal por diez años, acompañamiento institucional para gestionar créditos y solicitar garantías y, asistencia técnica y apoyo a proyectos de investigación, desarrollo e innovación, entre otros.

Se creó también una incubadora de proyectos tecnológicos, con el objetivo de detectar jóvenes con ideas de alto impacto, brindarles un espacio físico, opciones de financiamiento y una red de contactos para transformar los proyectos en empresas. Por otro lado, en conjunto con las empresas del sector, se desarrollaron programas de capacitación en testing (control de calidad), en los cuales el municipio cofinanció los cursos e identificó a los beneficiarios (Finquelievich, Feldman y Girolimo 2017). Desde allí se llevan adelante iniciativas para acompañar y asesorar a emprendedores, se gestionan distintos tipos de fondos de organismos nacionales para empresas de base tecnológica y pymes y, a través de la Agencia Nacional de Producción de Inversiones y Exportaciones, se incentiva a la generación de nuevos mercados.

En líneas generales, si bien en los inicios del Polo Informático la participación del municipio fue más bien accesoria, actualmente se observa un importante apoyo por parte del municipio al sector de software y servicios informáticos local, entendiendo a este como estratégico para la ciudad. A este respecto, a partir del año 2011, el municipio promovió un proceso de planificación estratégica participativa mediante el impulso de espacios de concertación, a fin de delinear el desarrollo futuro de Tandil. Se planteó como objetivo crear durante el período 2011-2015 un conjunto de planes estratégicos sectoriales de la ciudad. Para esto, se invitó a los

7 Son beneficios tributarios, por un plazo de diez años para las empresas que desarrollen software. La exención impositiva en ingresos brutos, inmobiliario y sellos puede ser, según los casos, del $70 \%$ o el $100 \%$ de la facturación de la empresa, priorizando las actividades de diseño, producción, implementación y puesta a punto de los sistemas de software desarrollados y su documentación técnica asociada. 
distintos actores a participar en la elaboración y posterior ejecución de las políticas públicas que los involucran.

Por otro lado, a partir del año 2018, se comenzó a trabajar en conjunto con CEPIT en un Plan Estratégico de Modernización del Municipio, a fin de actualizar los procesos administrativos y comunicacionales con tecnología. Se desarrollaron también diferentes actividades con los centros de investigación de la Unicen (Pladema e Isistan), para desarrollar, por ejemplo, un sistema de videovigilancia y, el proyecto financiado por Conicet sobre ciudades inteligentes, que suma también a las empresas del sector software y servicios informáticos, con el objetivo de construir en conjunto aplicaciones móviles o web para la ciudad (Finquelievich 2018).

Como resultado, hoy Tandil cuenta con cinco planes estratégicos sectoriales, de los cuales deben surgir proyectos concretos y consensuados para ser ejecutados por los propios actores. Dichos planes representan a los sectores de turismo, agroindustria, comercio, software y biotecnología, considerados estos, como los sectores principales de la ciudad.

En el próximo apartado se presentarán las principales características del Plan Estratégico del Software y Servicios Informáticos, cuya etapa de implementación comenzó en el año 2015 y tiene una proyección hasta el año 2023, coincidiendo con el año del bicentenario de Tandil.

\section{PLAN ESTRATÉGICO DEL SOFTWARE Y SERVICIOS INFORMÁTICOS DE TANDIL}

En marzo del año 2015, el Municipio convocó a la CEPIT y a sus asociados $y$, a los actores de la comunidad IT local que quisieran participar, para el armado del Plan Estratégico del Software y Servicios Informáticos de Tandil (PESSIT) para el período 2015-2023.

Para la elaboración del plan se realizaron diversos talleres de los cuales formaron parte distintos profesionales y empresarios del sector, coordinados en conjunto por la Unicen, la CEPIT y el Municipio. En los encuentros se lograron definir los acuerdos básicos relacionados al escenario futuro, las estrategias colectivas, los proyectos estructuradores claves y la organización para su ejecución.

Como parte del plan, cconsensuaron una visión y una misión que identifica al sector de software y servicios informáticos local. Al respecto, el conjunto de empresas manifestó que a largo plazo quieren "ser proveedores innovadores globales de productos y servicios informáticos de alta calidad 
a través de un consolidado ecosistema que integra los sectores público, privado, científico y tecnológico" (Municipio de Tandil y CEPIT 2015: 1).

Esta manifestación, que indica la meta en el largo plazo del sector, confirma la fuerte proyección exportadora que tienen las empresas, quedando claro que más allá de ser una iniciativa de desarrollo local creado por la Unicen, el Polo Informático constituye un importante negocio internacional. En este sentido, claramente el objetivo de las empresas apunta a la profundización internacional de sus vinculaciones de negocios por sobre lo local. No obstante, esto podría traducirse en una plataforma de vinculación con la estrategia de desarrollo de la ciudad y, a su vez, de la proyección regional de la Unicen.

Asimismo, el conjunto de empresas reconoce como un objetivo en común el fortalecimiento del entramado público-privado, académicocientífico y tecnológico, entendiendo que esta sinergia es clave para su crecimiento y posicionamiento tanto a nivel local como internacional.

En este marco, el PESSIT es un conjunto de lineamientos estratégicos planteados para el desarrollo competitivo del sector con proyección hacia el año 2023. Propone el diseño y la ejecución de las políticas públicas locales y las acciones necesarias para fortalecer el sector de software y servicios informáticos y generar un centro tecnológico de excelencia en la ciudad.

Como objetivos centrales del PESSIT fueron planteados el fortalecimiento institucional, las mejoras a nivel infraestructura, el soporte brindado a las empresas y los recursos humanos capacitados.

Es importante destacar que la escasez de recursos humanos calificados es un factor de importancia considerable por las empresas del sector de software y servicios informáticos. Es notorio el crecimiento producido en la demanda de soluciones informáticas en comparación a la matrícula académica y graduados de carreras referidas al sector. Esta situación provoca que la captación de recursos humanos por partes de las empresas tienda a extenderse a niveles inferiores de formación, proporcionando al sector recursos humanos menos capacitados. $\mathrm{Al}$ respecto, el PESSIT propone una mayor articulación con la Unicen a fin de capacitar recursos humanos.

Por otro lado, con el objetivo de lograr la mejora competitiva de las empresas del Polo Informático, se definieron cuatro estrategias transversales a fin de mejorar la comunicación interna y externa del sector de software y servicios informáticos, permitiendo una mejor articulación entre los 
sectores productivos e institucionales. En este sentido una de las estrategias es el desarrollo de esquemas formales de comunicación con todos los sectores que permitan planificar proyectos conjuntos. En tanto que las otras tres estrategias están orientadas a la búsqueda de una mayor eficiencia y eficacia intrasectorial, apuntando como ejes al fortalecimiento del capital humano, al impulso, desarrollo y gestión del sector y al soporte y apoyo a las empresas.

El desarrollo del plan estratégico permitió organizar y delinear el trabajo conjunto entre la Unicen, el municipio y la CEPIT con respecto al Polo Informático. A partir de entonces, la CEPIT concentró sus esfuerzos en concretar lo planteado en el PESSIT. En el año 2018 fue formalizado un directorio conformado por el Intendente, el rector de la Unicen y el presidente de CEPIT, que funcionará como órgano deliberativo para la toma de decisiones y la ejecución de las políticas vinculadas al PESSIT en la búsqueda del crecimiento y fortalecimiento del sector (ENE 2018a).

El PESSIT, creado con el objetivo de apoyar el crecimiento económico y la creación de empleo en la ciudad de Tandil, busca generar proyectos de inversión y dinamismo de la economía local y mejorar la competitividad del sector de software y servicios informáticos. En este marco, con su lista de proyectos prioritarios enfatiza la importancia de la productividad, la sustentabilidad, la equidad, la construcción de capital social y del fortalecimiento del capital humano.

Como se puede inferir, mediante la elaboración y ejecución en conjunto del PESSIT, como una base a partir de la cual se desarrollarán las políticas públicas de fomento al sector y al crecimiento económico del territorio, se asienta una dinámica de trabajo formal entre los tres actores centrales del Polo Informático, resaltando los beneficios sinérgicos provenientes de la relación triangular que se plantea en la teoría del triángulo de Sábato y la triple hélice (Sábato y Botana 1970, Sábato 1975, Etzkowitz y Leydesdorff 1997, Etzkowitz 2002).

La interacción entre los tres actores o tres vértices del triángulo se entiende a partir de las capacidades de cada sector. En este caso, el gobierno local se presenta como un articulador que propone una estrategia de acción deliberada para planificar e implementar las políticas públicas necesarias que acompañen al desarrollo del sector trabajando en conjunto con la Unicen, representante del complejo científico y tecnológico, y la CEPIT y el conjunto de empresas del sector, como la estructura productiva que aporta con su capacidad emprendedora (Del Bello 2015). En efecto, este tipo de iniciativas reconoce el funcionamiento sistémico entre todos 
los elementos en un accionar articulado, y deja asentado que el resultado depende del éxito de esa sinergia.

\section{EL SECTOR DE SOFTWARE Y SERVICIOS INFORMÁTICOS EN TANDIL}

El sector de software y servicios informáticos resulta estratégico para el crecimiento económico y el desarrollo social de un país. Constituye un importante generador de empleo de calidad y sus productos tienen la capacidad de generar efectos positivos de manera transversal en los procesos productivos de casi todos los sectores de la economía, impulsando, de esta manera, la competitividad del territorio junto con nuevas posibilidades de inserción en las cadenas globales de valor. Es decir, permite dinamizar el escalonamiento dentro de las cadenas globales de valor, el cual debe ser entendido como un proceso amplio de fortalecimiento de vínculos hacia atrás y hacia delante y de creación de canales hacia nuevos mercados (Staritz, Gereffi y Cattaneo 2011).

En efecto, un aspecto fundamental del sector en su impacto para el desarrollo local es su alto potencial exportador. Mientras que durante los primeros ańos del Polo Informático la mayor parte de la producción local se destinaba al mercado nacional, actualmente el mercado externo absorbe más del 70\% de lo que se produce en Tandil (Dabós 2018). Según Aníbal Carmona, Presidente de la Cámara de la Industria Argentina del Software y Servicios Informáticos (CESSI), el Polo Informático de Tandil cuenta con todo lo necesario para continuar progresando y poder transformar digitalmente el entramado productivo de la ciudad en los próximos veinticinco años (ENE 2017).

Como se señaló anteriormente, el Polo está compuesto por alrededor de 60 empresas, de las cuales más de un $50 \%$ son pymes producto de emprendimientos locales ${ }^{8}$ que emplean en promedio, entre 20 y 25 empleados cada una, seguidas por empresas nacionales e internacionales con sedes o filiales en la ciudad (Finquelievich 2018).

Las empresas deciden radicarse en Tandil debido a que encuentran recursos humanos altamente formados por la Unicen, un estilo de vida confortable y relajado, buena conectividad y cercanía respecto a la Ciudad Autónoma de Buenos Aires (CABA). Estos factores que se presentan como beneficios actuales para instalar las sedes, fueron también los que se priorización durante los inicios del Polo. Al respecto, el Polo Informático

8 De las 50 empresas asociadas a la CEPIT, 32 son de origen tandilense (incluidas las de zonas aledañas). 
logro impulsarse, justamente mediante la radicación de este tipo de empresas.

Según un informe de la CEPIT (2018), realizado en base a un relevamiento de 37 empresas, entre los ańos 2007 y 2017 fueron creadas 21 empresas en Tandil. En tanto que cinco nuevas empresas se radicaron en la ciudad. El número de empresas del Polo Informático fue en aumento constante desde su creación. Sin embargo, es importante destacar que en el camino se realizaron varios acuerdos de absorción y/o fusiones, como también existieron empresas que no prosperaron y otras que se marcharon de la ciudad porque aparecieron nuevos negocios.

Dentro del conjunto de compañías radicadas, las empresas más grandes en cuanto a cantidad de empleados son:Tivit, empresa brasilera que tiene más de 20.000 empleados y es líder en servicios integrados de tecnología en América Latina; Infor, multinacional con más de 15.000 empleados, la proveedora de tecnología privada más grande del mundo; Globantcon casi 6.000 empleados a nivel mundial; y Grupo ASSA (GA), firma argentina con más de 1.400 empleados dedicada a la consultoría y externalización de servicios tecnológicos.

A nivel local, las firmas con más cantidad de empleados son Globant, GA y Spark Digital, las dos primeras con un promedio de casi 150 empleados en Tandil. En tanto que las empresas que más personal contratan actualmente son Globant, GA, Technisys, EDSA y Spark Digital, las dos últimas de origen tandilense.

De acuerdo con CEPIT (2018), alrededor de un 59\% de las firmas tienen entre 1 y 10 empleados, un $20 \%$ tienen entre 11 y 30 , y un $11 \%$ entre 31 y 90 empleados, mientras que solo el 5\% cuentan con más de 100 empleados, a la vez que también un 5\% son empresas unipersonales y/o profesionales independientes. Del total de empresas relevadas a fines del año 2017, el 90\% manifestó la necesidad de contratar más empleados en un corto y mediano plazo, lo cual implicaría aproximadamente 476 nuevos cargos por cubrir.

Según la investigación realizada por Andrea Rivero (2018), el 90\% de los empleados de este tipo de empresas forma parte del área core (desarrollo), que estaría compuesta por el área técnica y en general con un plan de carrera. El resto es el equipo de soporte o gestión, el cual permite que el

9 El concepto de core business puede traducirse como la actividad principal o el negocio principal de una empresa. El core business es la razón de ser de la compañía, aquello por lo cual se crea y en lo que se va a generar el máximo valor ańadido. 
área core funcione. Por otro lado, Paula Dabós (2018) sostiene que desde el ańo 2011 se han desarrollado en Tandil varias micro empresas con entre 3 y 10 personas, que atienden a distintos clientes o realizan alguna porción de un proyecto más grande. Al trabajar en algún producto muy puntual, como por ejemplo alguna aplicación mobile, no requieren de una gran estructura.

Los servicios y productos que desarrollan las empresas varían desde integración de software y hardware hasta comercialización de productos informáticos y servicios de programación para terceros (Municipio de Tandil y CEPIT 2015). Existen también, empresas especializadas en banca, laboratorios, en gobierno, en sistemas móviles para usuarios finales, diseńo web, aplicaciones de entretenimiento, juegos, campañas publicitarias, software de gestión y de facturación, entre otros.

Según el relevamiento citado, las principales actividades productivas de las empresas son: diseño y desarrollo de software y de productos, mantenimiento de software, soporte a usuario, productos ERP, CRM, E-Commerce, big data y business intelligence, cloud computing, infraestructura y telecomunicaciones, entre otros (CEPIT 2018).

La mayoría de las empresas del sector software y servicios informáticos local efectúan desarrollos a medida, diferenciándose entre las que realizan productos con contenido de investigación y desarrollo y aquellas en las que prevalece un tipo de innovación incremental o adaptación local de los productos. Este último grupo realiza innovaciones comerciales mediante la incorporación de algo nuevo al sistema productivo, pero la mayor parte de las innovaciones no son radicales, sino pequeños cambios y mejoras.

Sucede que para las innovaciones radicales se necesita realizar una importante inversión y muchas veces los financiamientos disponibles son específicos y difíciles de conseguir. Teniendo en cuenta que en Tandil la mayoría de empresas son pymes, se desarrollan principalmente innovaciones incrementales, debido a que los costos son más accesibles. Por otro lado, en Tandil no existe competencia directa entre clientes y proyectos, dado que las empresas se especializan en distintos nichos, generando una importante cohesión entre las mismas dentro del Polo Informático local (Rivero 2018).

Las actividades de comercialización a las cuales se dedican las empresas del sector de software y servicios informáticos en Tandil son muy variadas, siendo los sectores salud, servicios financieros, transporte y logística, administración pública, energía y turismo los más populares. En el Gráfico 
1 se detallan todos los sectores en los cuales comercializan las empresas relevadas por la CEPIT (2018).

Gráfico 1: Sectores de comercialización del software y servicios informáticos de Tandil, 2017

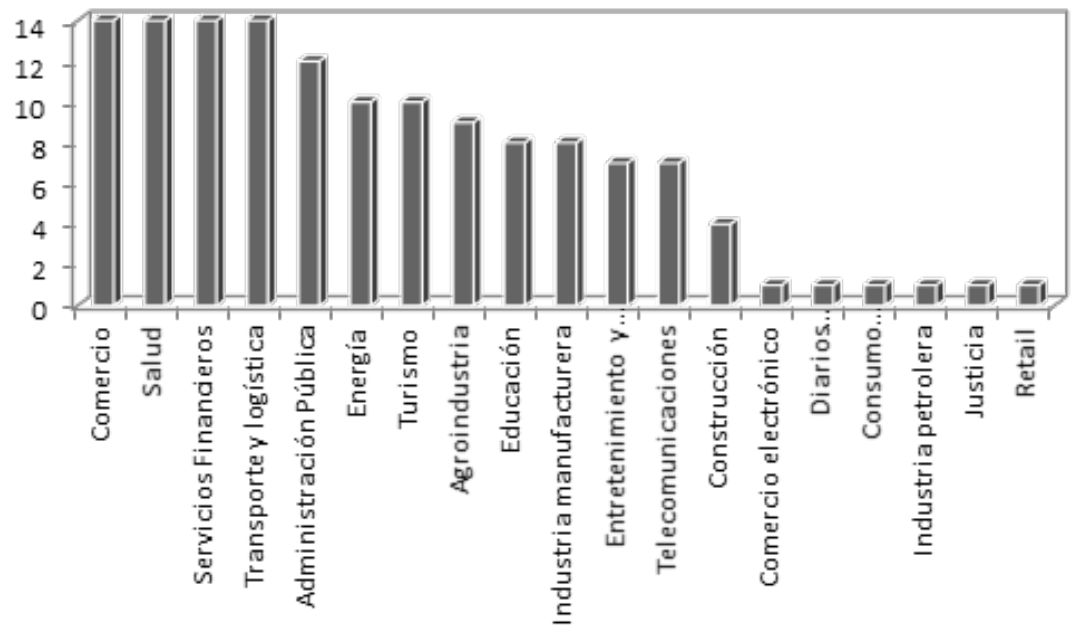

Fuente: CEPIT (2018).

Si bien es posible observar una mayor tendencia de las empresas hacia los sectores de salud, finanzas, transporte, administración pública, energía y turismo, el sector de software y servicios informáticos local se caracteriza por un importante grado de diversificación hacia distintos mercados y clientes de todos los rubros.

$\mathrm{Al}$ igual que lo analizado previamente en materia de empleo, el observar las firmas por nivel de facturación se destaca una mayoritaria presencia de pymes dentro del Polo Informático local, superando el 90\% del total de empresas (CEPIT 2018). En cuanto a su inserción comercial, las empresas del sector de software y servicios informáticos local tienen una importante orientación exportadora, lo cual se evidencia en que el $70 \%$ de lo que se produce en Tandil es exportado y, de acuerdo a referentes del sector, el objetivo a mediano plazo es continuar aumentando esta proporción (Salvatierra 2018, Dabós 2018). Como meta, el conjunto de empresas apunta a que el Polo Informático de Tandil sea reconocido como un proveedor global innovador de software y servicios informáticos.

Actualmente el sector de software y servicios informáticos exporta a distintos países de África, Asia, América Central, Europa y a gran parte de los países de América del Sur y América del Norte. En el Gráfico 2 se presentan los principales destinos de exportación. Estados 
Unidos se presenta como el principal destino de exportación del sector de software y servicios informáticos de Tandil. Asimismo, esta tendencia a nivel local coincide con lo que sucede a nivel nacional. Esto se explica en parte, debido a que muchas empresas se radican en Estados Unidos porque a diferencia de otros mercados, no retiene impuesto a la renta por las importaciones de servicios (sí lo hace en el caso de las regalías), por lo tanto, en las exportaciones se incluye el comercio intrafirma de las empresas exportadoras.

Gráfico 2: Principales países destinos de exportación del sector de

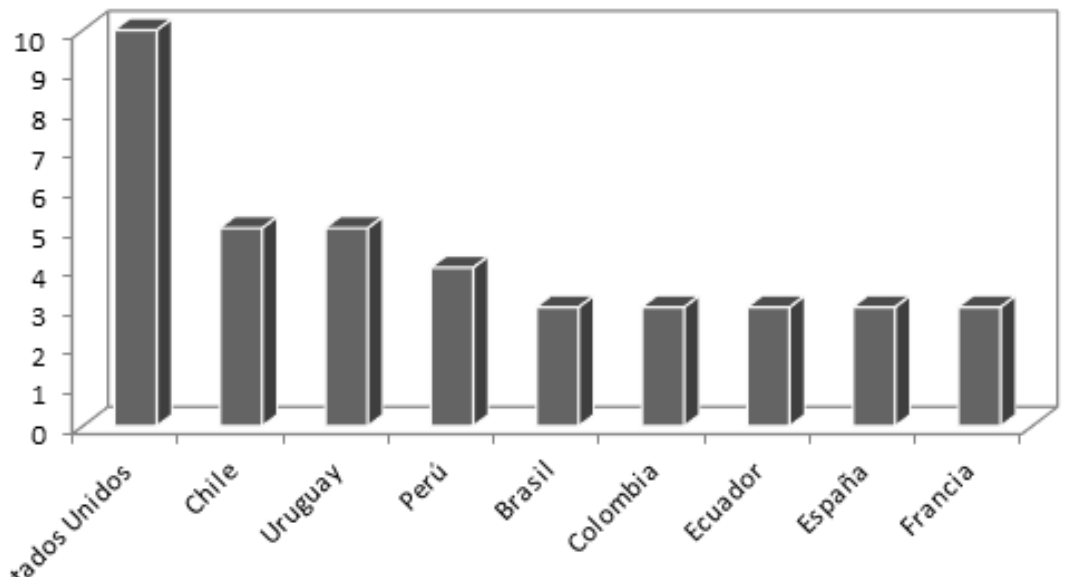

Fuente: CEPIT (2018).

Es importante destacar que Brasil, a diferencia de lo que sucede con las exportaciones de bienes y servicios tradicionales, se presenta como un mercado de difícil acceso debido al régimen impositivo específico ${ }^{10}$. En efecto, según Salvatierra (2018), una de las estrategias que están desarrollando las firmas locales para ingresar en el mercado brasilero es la instalación de una sede en el país vecino.

Por último, resulta interesante revisar la situación de la fuerza laboral del sector de software y servicios informáticos local. Es importante destacar que no existe ningún convenio homologado por el Ministerio de Trabajo de la Nación (CESSI 2018), con lo cual, sin ser limitadas por un convenio colectivo de trabajo, las empresas cuentan con libertad de gestión. No obstante, si bien esta industria se caracteriza por una baja sindicalización tanto a nivel nacional como local, existe un creciente debate sobre la distribución de los ingresos en la industria del software (ENE 2018b).

10 En 2019, entró en vigor el acuerdo firmado entre Argentina y Brasil que elimina la doble imposición tributaria que permitirá reducir entre un 10\% y 15\% de los costos de exportación. 
La agrupación Trabajadores Informáticos de Tandil (TIT) logró en julio del año 2018 la formalización como delegación regional de la Asociación Gremial de Computación ${ }^{11}$. De esta manera, la TIT adquirió legitimidad para dialogar sobre condiciones de trabajo y cuestiones salariales frente a otros organismos.

Las empresas del sector de software y servicios informáticos en general ofrecen empleo estable, registrado y con otros beneficios como, por ejemplo, la flexibilidad para cumplir el horario o clases de inglés durante la jornada laboral o de manera complementaria. En tanto que dentro del sector las categorías y roles de los empleados son muy disimiles, dificultando la generalidad de los puestos de trabajo y por ende de los salarios percibidos. Por un lado, se da una categorización basada en la experiencia adquirida con respecto a una tecnología en particular (junior, semisenior, senior) y, en paralelo, existen distintos roles enfocados más a tareas de gestión (líder de equipo, líder de proyecto, etc.), pudiendo el empleado tener un tipo de categoría y a su vez un tipo de rol.

Un aproximado de los salarios que reciben los empleados del sector local según la categoría, serían los siguientes (valores mensuales en bruto y en dólares): para un técnico con categoría junior (técnico sin experiencia previa) los sueldos pueden rondar entre los US\$ 455 y los US\$ 680. Un técnico semisenior (técnico con algo de experiencia) entre los US\$ 780 y los US\$ 965. En tanto que un senior (cinco años de experiencia aproximadamente) ronda entre los 1.040 y 1.300 y por ejemplo un senior java o mobile puede estar ganando US\$ 1.820. Mientras que los empleados freelance pueden alcanzar una suma de US\$ 4.000 y algunos llegan hasta los US\$ $8.000^{12}$.

De lo expuesto hasta aquí y a modo de síntesis, se puede señalar que el Polo Informático de Tandil representa un sector que se destaca por poseer un significativo potencial de crecimiento en el espacio económico local y como generador de exportaciones de servicios y productos diversificados. Desde allí, que actualmente representa un sector estratégico para el desarrollo económico, social y territorial de la ciudad, cuya importancia es reconocida por los principales actores locales. Asimismo, la existencia del polo resulta clave para el desarrollo competitivo del sector y para promover al mismo tiempo la vinculación comercial internacional de Tandil.

11 Entidad nacional que se constituye como referencia de los trabajadores informáticos desde la década de los noventa y que puja por oficializarse como el sindicato del sector en el país.

12 Datos aproximados armados en base a datos publicados en julio del 2018 por OPSSI y respuestas de encuestas realizadas de manera anónima a empleados del sector local. 


\section{IMPACTO SOCIOECONÓMICO DEL SECTOR DE SOFTWARE Y SERVICIOS INFORMÁTICOS A NIVEL LOCAL}

El desarrollo del Polo Informático constituyó una forma innovadora de pensar y diseńar el crecimiento del sector de software y servicios informáticos y, en consecuencia, una herramienta central para avanzar en el desarrollo económico a nivel local y regional.

En este sentido, iniciativas como las del Polo se convierten en receptores de inversiones que ven en este ámbito un lugar de concentración de demanda. Además, se convierten también en socios inseparables de los procesos de regionalización, siendo alternativas de desarrollo para regiones alejadas de las ciudades centrales, como es justamente el caso de Tandil (Calvento et al. 2016).

Desde su conformación el Polo generó las condiciones necesarias para que se creen, radiquen y se fortalezcan empresas de base tecnológica. Asimismo, promovió el afianzamiento de la vinculación entre la Unicen y las empresas del sector de software y servicios informáticos, generando una mayor transferencia de tecnologías desde la comunidad universitaria al medio productivo, estimulando, a su vez, la creación de distintas start-ups y spin-offs, muchas de origen universitario (Ribas, Dabós y Rivero 2007), como es el caso de Media.Lab ${ }^{13}$ surgida a partir del trabajo del instituto de investigación Pladema de la universidad.

Para la ciudad, el Polo Informático es una importante fuente generadora de puestos de trabajo. Como se señaló anteriormente, en el año 2003 se calculaban 65 puestos de trabajo concebidos por el sector, para el año 2018 se calculan alrededor de 1.800, incluyendo a los centros y grupos de investigación dependientes de la Universidad. En conjunto los perfiles ocupacionales provienen de distintas disciplinas y facultades, asegurando un abanico de posibilidades de desarrollo profesional amplio a nivel local (ENE 2018c).

En cuanto al origen de los recursos humanos empleados por el sector, se estima que alrededor del $30 \%$ responde a gente oriunda de Tandil, mientras que el $60 \%$ estudió en la ciudad y el restante $10 \%$ responde a personas radicadas a partir de la presencia del Polo Informático (Salvatierra 2018).

13 Espacio multidisciplinario centrado en la investigación y desarrollo de aplicaciones de computación gráfica, realidad virtual e interacción humano-computadora. 
De esta manera, la presencia del Polo genera demanda para el mundo gastronómico, artístico, recreación y consumo, y demandas inmobiliarias de corto y mediano plazo. Asimismo, promociona la ciudad con su atractiva fórmula de conocimiento, calidad de vida y belleza natural. En particular, esto atrae a diversos profesionales de CABA que deciden radicarse junto a sus empresas. En conjunto, la presencia del Polo retiene a más del 85\% de la matrícula de ingeniería en sistemas de la Unicen, así como también motivó el regreso de profesionales que anteriormente habían migrado por falta de oportunidades laborales en Tandil, produciéndose el fenómeno opuesto al que se registraba durante los inicios de la década del 2000 (Ribas, Dabós y Rivero 2007).

En este marco, el desarrollo del Polo Informático se convierte en un factor dinamizador de la economía a nivel local, generando un efecto multiplicador característico de los agrupamientos de empresas nacidos en entornos denominados innovadores (Calvento et al. 2016). A su vez, el sector de software y servicios informáticos es en sí mismo un sector dinámico que además de generar y producir al interior de la industria del software, se expande al resto de las industrias, dado que estas últimas necesitan mucho del software en sus procesos, desde los recursos humanos hasta las líneas de producción.

Por otro lado, Carlos Pallotti ex Subsecretario de Servicios Tecnológicos y Productivos del Ministerio de Producción e impulsor del Plan 111 Mil, realizó algunas estimaciones generales del impacto que el Polo Informático estaría produciendo en la economía tandilense. Pallotti sostuvo que el total de personas empleadas por el sector generarían de manera directa y por efectos indirectos más de \$1.000.000.000 de ingresos netos en la economía general: en gastos en negocios; en pago de impuestos, tasas, alquileres; entre otros, lo cual permitiría que más de 11.000 personas pudieran recibir un ingreso mínimo superior a los $\$ 13.000$ (Taborga 2018).

En síntesis, el accionar del conjunto de empresas del Polo Informático de Tandil ha tenido un indiscutible impacto socioeconómico en la ciudad, transformando el perfil productivo territorial y abriendo oportunidades de inserción internacional en segmentos del mercado global sumamente competitivos.

\section{REFLEXIONES FINALES}

La hipótesis que motivó y guió el trabajo sostiene que la iniciativa de la Universidad Nacional del Centro de la Provincia de Buenos Aires para desarrollar el Polo Informático, con apoyo del municipio y del sector 
privado (en un principio empresas individuales y luego agrupadas bajo la figura de CEPIT), implicó la implementación de un conjunto de herramientas dinamizadoras que derivaron, desde lo sectorial, hacia una estrategia de desarrollo local y de vinculación internacional para el conjunto de la ciudad.

A partir de allí, y en función del análisis e interpretación de los hechos planteados a lo largo del artículo, es posible confirmar que actualmente el Polo Informático se destaca por ser uno de los sectores más dinámicos en su aporte al crecimiento productivo de la ciudad, cuyo potencial de desarrollo para la expansión local y su inserción internacional es superior al resto de la economía de la región.

En este sentido, no solo se desarrolló un mercado laboral creciente para que los estudiantes y graduados logren insertarse profesionalmente, sino que a su vez generó un ecosistema emprendedor para que se desarrollen empresas de base tecnológica en la ciudad, fortaleciendo la presencia de un sector clave en el devenir tecnoeconómico global. Asimismo, esta experiencia en torno a la conformación del polo generó una sinergia de trabajo positiva sin precedentes a nivel local entre la universidad, el sector privado y el municipio.

Es importante señalar que, siguiendo los datos del Instituto Nacional de Estadísticas y Censos (INDEC) y del Banco Mundial, en el año 2017 las exportaciones de servicios basados en el conocimiento de Argentina significaron alrededor del $0,3 \%$ del total de servicios basados en el conocimiento exportados en el mundo. Estos valores se encuentran en consonancia con la participación del país dentro del total de exportaciones mundiales de bienes y servicios, que, según el Banco Mundial, fue aproximadamente del $0,3 \%$. Por su parte, debe destacarse que en las exportaciones mundiales del sector de software y servicios informáticos Argentina duplicó su participación, alcanzando el 0,7\% del total, lo cual refleja un posicionamiento competitivo, la potencialidad del sector y la acumulación de capacidades en términos relativos.

En efecto, el conjunto de evidencias analizadas permite señalar a la Argentina como uno de los líderes a nivel regional en la producción de software y servicios informáticos. Esta situación obliga a pensar y diseñar marcos específicos con los distintos países de la región para aprovechar esa mejor posición relativa y conseguir nuevos acuerdos comerciales para la inserción de América Latina a nivel global, es decir, aprovechar la participación de los distintos países en las cadenas globales de valor y 
desarrollar incentivos para que Argentina pueda insertarse en ellas mediante la provisión de software y servicios informáticos a sus socios regionales.

Resulta importante seńalar que el despliegue y consolidación del sector a nivel nacional fue propiciado por un contexto económico de recuperación productiva y crecimiento sostenido hasta 2011, pero un contexto de estancamiento desde entonces. Asimismo, se destacaron una serie de políticas públicas e instrumentos de promoción a nivel nacional, provincial y local que dieron marco a las condiciones favorables para su desarrollo.

En el caso de Tandil, el liderazgo de la Unicen fue fundamental para el desarrollo del Polo, visibilizando la importancia y la capacidad del sector público para llevar adelante este tipo de iniciativas. Es decir, la universidad constituyó el agente central de vinculación tecnológica del sistema de innovación (de preeminencia pública) hacia el sector privado y, especialmente, con implicancias para la comunidad por medio de su posterior articulación con la estrategia de desarrollo local.

Como resultado, la universidad tuvo un rol clave como dinamizadora de los negocios locales e internacionales y como impulsora de la asociación de elementos y actores para la identificación de un sistema local de innovación. Con esta finalidad, sentó las bases del Polo Informático, generando y desarrollando las condiciones necesarias para que se establezcan las empresas, formando los recursos humanos necesarios y desarrollando políticas activas de vinculación.

La Unicen reconoció las demandas del contexto y brindó una respuesta efectiva a las mismas. Desde los inicios del polo los esfuerzos de la institución con el desarrollo económico y social de la región se vio reflejado en las diversas actividades llevadas adelante para vincular y transferir actividades científicas y tecnológicas a la comunidad y a los sectores productivos. No obstante, la transferencia tecnológica y la vinculación del sector de software y servicios informáticos se eleva como un desafío central que deberán abordar en conjunto la universidad, la CEPIT y el municipio, no solo pensando en el crecimiento y posicionamiento de las empresas del sector en sí, sino también pensando en el desarrollo productivo y social de la ciudad y la región en un nivel más amplio, atendiendo a demandas de la comunidad y sujetas al bienestar general de la población.

A lo largo del artículo se señaló que la creación de la CEPIT constituyó un hecho central para la consolidación del sector. Desde su creación ha evolucionado organizativa e institucionalmente para liderar el Polo Informático, y actualmente representa el principal canal de comunicación 
interna entre los miembros y respecto a la dinámica de trabajo con la Unicen y el municipio. Con lo cual, es posible afirmar que, debido a su importancia y relevancia creciente en la estructura productiva local, en la actualidad tiene incidencia real en el diseño y formulación de políticas públicas locales vinculadas al sector.

Desde allí, se debe explicitar la necesidad de aumentar la producción de datos a nivel local, para lo cual se propone que, mediante la articulación de los distintos actores, se pueda constituir un laboratorio de datos y de seguimiento del sector de software y servicios informáticos en la región.

Por su parte, el municipio ha logrado acompañar el proceso de creación y consolidación del Polo Informático con políticas específicas para el sector, como las políticas fiscales a nivel local apoyadas con recursos institucionales y financieros. De hecho, la mencionada planificación estratégica de la ciudad confirma la importancia del sector para la gestión de gobierno que considera la centralidad del software y servicios informáticos para el desarrollo de Tandil.

En un contexto en el cual el rol de las ciudades adquiere cada vez más importancia en la constitución de redes globales, es importante valorar el hecho de que el gobierno local apoye las iniciativas en torno a las oportunidades que representan las nuevas tecnologías para la inserción más eficiente de los diversos territorios en la economía global. En este sentido, el caso de estudio abordado se presenta como un significativo ejemplo debido a las señaladas capacidades para generar y consolidar redes que permiten la interacción y el flujo de conocimiento entre los distintos actores que forman parte del territorio $\mathrm{y}$, asimismo, aprovechar las oportunidades para fortalecer la vinculación de lo local con el plano internacional.

En este sentido, se espera que el trabajo contribuya a difundir el caso de Tandil como una experiencia exitosa de desarrollo, centrada en los negocios internacionales y la sinergia entre actores del territorio, y por lo tanto pueda inspirar políticas públicas e iniciativas de asociación desde el sector privado. 


\section{REFERENCIAS}

Alburquerque, F. (2002). Marco conceptual y estrategia para el desarrollo local. Recife: BNDES/PNUD.

Becattini, G. (2002). Del distrito industrial Marshalliano a la teoría del distrito contemporánea, una breve reconstrucción crítica. Investigaciones Regionales. Asociación española de ciencia regional, 1, $9-32$.

. (2004). Industrial districts: A new approach to industrial change. Cheltenham: Edward Elgar Publishing.

Blanco, E. (2016). Gran futuro en Argentina para los servicios basados en el conocimiento. Disponible en https://portinos.com/31780/gran-futuroen-argentina-para-los-servicios-basados-en-el-conocimiento [22-062018].

Calvento, M., Araya, J., Di Meglio, F., Nicolao, J., Herrero, M., Colombo, S., Lorenzo, N., Loray, R., Rolandi, M., De Angelis, I., Carcedo, J. y Mutti, A. (2016). Diagnóstico de la gestión internacional del Municipio de Tandil. Propuestas para su fortalecimiento. Tandil: UNIVEN-CEIPILANPCyT.

Capellán, N. (2016). Ciudades inteligentes. El aporte de las TIC a la comunidad. Casos testigo y la visión del sector privado. Buenos Aires: Colección CICOMRA.

Centro para el Desarrollo Tecnológico Industrial (CDTI) (2007). La deslocalización empresarial en España. Cuadernos CDTI de la innovación tecnológica. Disponible en: www.cdti.es/recursos/publicaciones/ archivos/21623_210210200714555.pdf [10-02-2019].

Cámara de Empresas del Polo Informático de Tandil (CEPIT) (2017). Monitor estadístico 2017. Tandil: Cámara de Empresas del Polo Informático de Tandil.

. (2018). Plan estratégico 2015-2023. Tandil: Cámara de Empresas del Polo Informático de Tandil.

Cámara de Empresas de Software y Servicios Informáticos (CESSI) (2018). Reporte anual sobre el Sector de Software y Servicios Informáticos de la 
República Argentina. Reporte año 2017. Tandil: Cámara de Empresas de Software y Servicios Informáticos.

Cravacuore, D. (2006). La articulación de actores para el desarrollo local. En Adriana Rofman (Ed.), Desarrollo Local. Una revisión critica del debate. Buenos Aires: Espacio.

Dabós, P. (2018). Entrevista. Directora y socia fundadora de la empresa Bee Real e Integrante de CEPIT. Octubre, 2018.

Dabós, G., Rivero, A. y Ribas, F. (2011). Mecanismos institucionales para la vinculación tecnológica: nuevos roles de la Universidad innovadora. En Rébori, A., Dabós, G. y D’Annunzio, C. (Eds.). Conocimiento, Innovación y Entrepreneurship. El rol de la Unicen y su impacto en el desarrollo regional. Tandil: Unicen.

De Angelis, I. (2017). Políticas de ciencia y tecnología para la producción industrial: modelos de desarrollo y sistemas de innovación en Argentina. Revista de Gestión Pública, 6 (2), 247-281.

De Angelis, I y Lorenzo, N. (2016). El perfil de participación internacional de Tandil: características y reflexiones sobre variaciones en su desempeño (2011-2015). En Calvento, M. et al. (Eds.). Diagnóstico de la gestión internacional del Municipio de Tandil. Propuestas para su fortalecimiento. Tandil: Unicen-CEIPIL-ANPCyT.

Del Bello, J. C. (2015). Autonomía y dependencia tecnológica. Seminario PLACTED- MINCyT-CITECDE. Seminario de Ciencia y Tecnología en el Pensamiento de Jorge Sabato, Oscar Varasavsky y Amílcar Herrera. Bariloche: Centro de Estudios en Ciencia, Tecnología, Cultura y Desarrollo.

Enfoque de Negocios (ENE) (2017). Transformación digital en la cuarta revolución industrial. Disponible en http://www.enfoquedenegocios. com.ar/2017/04/transformacion-digital-la-cuarta-revolucionindustrial/ [04-12-2018].

Disponible en http://www.enfoquedenegocios.com.ar/2018/05/ Municipio-cepit-y-unicen-firmaron-acuerdo-para-politicas-sobresoftware/ [04-12-2018]. 
Disponible en http://www.enfoquedenegocios.com.ar/2018/06/ agremiacion-el-camino-de-los-trabajadores-informaticos-de-tandilante-la-desregulacion-laboral/ [07-12-2018].

(2018c). La crisis universitaria y efectos sobre la industria del software local. Disponible en http://www.enfoquedenegocios.com. ar/2018/09/la-crisis-universitaria-amenaza-con-golpear-al-sectorlocal-con-mas-proyeccion/ [07-12-2018].

Etzkowitz, H. (2002). La triple hélice: universidad, industria y gobierno. Implicaciones para las politicas y la evaluación. Disponible en http:// www.sivu.edu.mx/portal/noticias/2009/VinculacionLatriplehelice.pdf [18-01-2018].

Etzkowitz, H. y Leydesdorff, L.A. (1997). Universities and the global knowledge economy: A triple helix of university-industry-Government relations. Londres: Pinter.

Fernandez-Stark, K., Bamber, P. y Gereffi, G. (2011). The offshore services value chain: upgrading trajectories in developing countries. International Journal of Technological Learning, Innovation and Development, 4 (1-3), 206-234.

Finquelievich, S. (2018). TIC e innovación productiva. Políticas públicas para el desarrollo local: Presente y futuros posibles. Buenos Aires: Teseopress.

Finquelievich, S., Feldman, P. y Girolimo, U. (2017). Tandil: innovación y desarrollo local. Cuaderno Urbano. Espacio, Cultura, Sociedad, 22 (22), 133-155.

Gajst, N. (2012). La construcción de las políticas públicas dirigidas al sector software y servicios informáticos argentino (2003-2010). Ponencia presentada en la VI Conferencia ACORN-REDECOM, 17 y 18 de mayo, Valparaíso, Chile.

Gayá, R. (2016). Fortalecimiento de los servicios basados en el conocimiento en Argentina. Disponible en https://www.produccion.gob.ar/wp-content/ uploads/2016/08/Redlas-Romina-Gaya.pdf [11-07-2018].

Jensen, P. (2009). A learning perspective on the offshoring of advance service. Journal of International Management (15), 181-193. 
Kraemer, K. L., Linden, G. y Dedrick, J. (2011). Capturing value in global networks: Apple's iPad and iPhone. Disponible en http:// economiadeservicos.com/wp-content/uploads/2017/04/value_ipad_ iphone.pdf [12-02-2020].

López Bidone, E. (2008). El proceso de innovación tecnológico y la glocalización de las actividades productivas: El parque tecnológico de la Universidad nacional del centro como caso de estudio (2003-2007) (Tesis de grado), Universidad Nacional del Centro de la Provincia de Buenos Aires, Tandil, Argentina.

López, A, Niembro, A. y Ramos, D. (2011). Cadenas globales de valor en el sector servicios: Estrategias empresarias e inserción de los países de América Latina. Integración y Comercio, 15 (32), 57-68.

Ministerio de Economía de la Provincia de Buenos Aires (MEPBA) (2014). Programación del desarrollo territorial. Diagnóstico preliminar y líneas para la discusión. Buenos Aires: Ministerio de Economía de la Provincia de Buenos Aires.

Municipio de Tandil y Cámara de Empresas del Polo Informático de Tandil (CEPIT) (2015). Plan estratégico del software y servicios informáticos de Tandil 2015-2023. Lineamientos estratégicos base para el desarrollo competitivo del sector del software y servicios informáticos del Municipio de Tandil. Tandil: Municipio de Tandil y CEPIT.

Observatorio Permanente de la Industria de Software y Servicios Informáticos (OPSSI) (2018). Reporte anual sobre el Sector de Software y Servicios Informáticos de la República Argentina. Buenos Aires: Cámara de la Industria Argentina del Software.

Rébori, A., D’Annunzio, C. y Dabós, G. (2011). Creación de nuevas empresas de base tecnológica: estructuras de apoyo a spin-offs de origen universitario. En Rébori, A., Dabós, G. y D’Annunzio, C. (Eds.), Conocimiento, innovación y entrepreneurship. El rol de la Unicen y su impacto en el desarrollo regional. Tandil: Centro de Estudios en Administración, Unicen.

Ribas, F., Dabós, G. y Rivero, A. (2007). Impacto de las políticas institucionales en la generación de empleo: Las Universidades innovadoras en sectores de alta tecnología. Anales de la Red PyMEs Mercosur, 12, 1-20. 
Rivero, A. (2018). Entrevista. Investigadora del Centro de Estudios en Administración de la Facultad de Ciencias Económicas de la Universidad Nacional del Centro de la Provincia de Buenos Aires. Septiembre, 2018.

Sábato, J. (1975). El pensamiento latinoamericano en la problemática ciencia-tecnología-desarrollo-dependencia. Buenos Aires: Paidós.

Sábato, J. y Botana, N. (1970). América Latina: Ciencia y tecnología en el desarrollo de la sociedad. Santiago de Chile: Editorial Universitaria.

Salvatierra, M. (2018). Entrevista. Gerente de Globant Tandil y Vicepresidente de CEPIT. Septiembre, 2018.

Scholvin, S. (2020). Global commodity chains, global value chains and global production networks. En Vivares, E. (Ed.), The Routledge Handbook to Global Political Economy. New York: Routledge.

Staritz, C., Gereffi, G. y Cattaneo, O. (2011). Shifting end markets and upgrading prospects in global value chains. International Journal of Technological Learning, Innovation and Development, 4 (1), 1-13.

Taborga, S. (2018). Historia, presente y futuro de la industria del software local [Audio en podcast]. Modo Tandil: conjunto de podcasts. Disponible en: https://ventostaborga.bandcamp.com/album/modo-tandil [20-122018].

Tamayo Plata, M. P. (2014). El fenómeno del offshoring. Ecos de Economía: A Latin American Journal of Applied Economics, 18 (38), 157-182.

Taylor, P. J. (2007). Cities, worlds cities, networks and globalization. GaWC Research Bulletin, 238.

Taylor, P. J. y Derudder, B. (2016). World city network: a global urban analysis. Nueva York: Routledge.

Velázquez, G., Mikkelsen, C., Linares, S. y Celemín, J. P. (2010). Calidad de vida en Argentina. Ranking del bienestar por departamentos. Tandil: Unicen.

Recibido: 12-02-2020

Aceptación de la versión final: 01-06-2020 